\title{
Microfiltration, Nano-filtration and Reverse Osmosis for the Removal of Toxins (LPS Endotoxins) from Wastewater
}

\author{
Guizani Mokhtar ${ }^{1,2 *}$ and Funamizu Naoyuki ${ }^{1}$
}

${ }^{1}$ Graduate School of Engineering, Hokkaido University, Japan

${ }^{2}$ Center for Sustainability Science, Hokkaido University, Japan

\begin{abstract}
Lipopolysaccharide (LPS) endotoxin, a bacterial byproduct abundantly present in wastewater, is more and more representing a major concern in wastewater treatment sector for the potential health risk it represents. It is, therefore, more urgent than before to protect consumers from contaminating their fresh potable water reserves with LPS endotoxin through aquifer replenishment using reclaimed wastewater or by supplying reclaimed wastewater as potable water. Membrane treatment is an alternative to activated sludge process and is the most commonly used to treat wastewater. Moreover, nano-filtration and reverse osmosis are the most advanced technologies used to treat wastewater to a potable level. Removal efficiency of LPS endotoxin using Membrane bioreactors (MBRs) and Nanofiltration (NF) and Reverse Osmosis (RO) is subject of this paper. It revealed that these advanced technologies could remove a significant amount of endotoxin. However, levels of concentration in the product water are still much higher than the one found in tap water and it is not advisable to supply this water directly to consumers. Further investigations are required to determine the best management practices for a safe supply of potable water from reclaimed wastewater.
\end{abstract}

Keywords: Lipo-polysaccharide (LPS); Microfiltration, Nanofiltration and Reverse Osmosis; Waterwater management

\section{Introduction}

There is an increasing demand for better quality of effluents from wastewater treatment processes. As assumed and with some incertitude, Lipo-polysaccharide (LPS) endotoxin materials, a bacterial byproduct [1] found in treated wastewater, are a potential threat for human health [2-5].

Knowing that it is almost not possible to control these chemicals in activated sludge process [6,7], endotoxin removal from secondary treated water is crucial and a preliminary step towards safe potable reuse of reclaimed wastewater as well as for fresh water resources contamination prevention. Several treatment alternatives can be considered for achieving this goal ranging from high-tech processes to simple natural, but effective, processes. Alternative removal technologies include membrane filtration (microfiltration, ultrafiltration, nano-filtration and reverse osmosis), soil treatment and coagulation, flocculation and sedimentation. In this paper we investigate the removal of organic matter showing endotoxicity found in the secondary effluents of wastewater treatment plants using membrane filters, including micro-filters, nano-filters and reverse osmosis.

Membrane bioreactors (MBRs), operated, as an alternative of conventional activated sludge system, have become an attractive water treatment alternative. MBR systems offer several advantages over conventional treatment systems. For instance, permeates of membranes with pore size of about $0.1 \mu \mathrm{m}$ to $0.4 \mu \mathrm{m}$ is of higher quality as compared to conventional effluent [8]. As matter of fact, MBRs are of interest wherever high quality effluent is required, as they do not replace only secondary clarifier but also replaces treatment steps like sand filtration and ultra-violet (UV) disinfection as well. Large molecules of endotoxin, which represent up to $80 \%$ of the total organic matter as stated in previous chapter, could be removed using MBR. However, effect of sludge retention time (SRT) on the molecule aggregation and therefore endotoxin removal is crucial and has to be evaluated.
Nano-filtration (NF) and reverse osmosis (RO) membranes have been increasingly used in wastewater reclamation in recent years for the production of clean water from sewage, benefiting due to their improvement in terms of energy consumption [2]. For the time being, they are almost employed in all wastewater treatment plants producing potable water quality. Efficiency of NF and RO in removing endotoxin is discussed in this article. Samples from feed water (MBR permeate) and NF/RO permeate will be taken and LPS endotoxin and dissolved organic matter (DOC) quantified.

Organic matter, showing endotoxicity in reclaimed wastewater, aggregates into molecules larger than $100 \mathrm{KDa}$ [8], which makes endotoxin removal from reclaimed water feasible using tight membranes. This can be enhanced by the domination of hydrophobic fractions of endotoxins [8]. In addition, ultra-filtration using membranes with about $10 \mathrm{KDa}$ nominal-molecular weight cut-off are routinely employed to get ultrapure water in laboratory systems and it is found in operation wherever endotoxins are not allowed to enter sterile equipment [9]. Furthermore, dead end ultra-filtration showed a good removal of endotoxin $(\mathrm{Rlog}=1)$. Such a property has potential utility in endotoxin removal from treated wastewater using membrane technology. Removal of endotoxin from reclaimed wastewater using ultra-filter, micro-filter, nano-filter and/or reverse osmosis is of great importance. To understand efficiency of these membranes in removing endotoxin from wastewater, a lab scale experiment using a set of MBR$\mathrm{NF} / \mathrm{RO}$ was operated. A two-stage filtration was used in this study

*Corresponding author: Guizani Mokhtar, Graduate School of Engineering, Hokkaido University, Japan, E-mail: g_mokh@yahoo.fr

Received August 01, 2012; Accepted September 05, 2012; Published September 12, 2012

Citation: Mokhtar G, Naoyuki F (2012) Microfiltration, Nano-filtration and Reverse Osmosis for the Removal of Toxins (LPS Endotoxins) from Wastewater. J Memb Sci Technol 2:118. doi:10.4172/2155-9589.1000118

Copyright: (c) 2012 Mokhtar G, et al. This is an open-access article distributed under the terms of the Creative Commons Attribution License, which permits unrestricted use, distribution, and reproduction in any medium, provided the original author and source are credited. 
during which water contaminated with endotoxin pass through 0.4 $\mu \mathrm{m}$ micro-filter membrane. After that, the membrane permeate run through either nano-filter or reverse osmosis.

\section{Materials and Methods}

\section{Water samples}

MBR: Because secondary effluent showed toxicity, we focused on the treatment capacity of MBR as an alternative to the activated sludge process. Membranes at longer sludge retention time (SRTs) are expected to have better biodegradation of organic matter and removal of toxic compounds as compared to the activated sludge process. In this study, three types of MBRs with different SRTs were examined.

Influent of the primary sedimentation basin of a domestic wastewater treatment plant in Sapporo was used as a feed for three types of MBRs, operated in parallel in this study as an alternative to conventional activated sludge system (Figure 1). MBR18 $($ SRT $=18)$ is baffled submerged MBR where the reactor is divided into two zones by the baffles. The inner zone is kept aerobic due to continuous aeration. In the outer zone, aerobic and anoxic conditions are alternatively created at a constant interval. The MBR18 used in this study was equipped with $6.8 \mathrm{~m}^{2}$ flat-sheet type of micro-filtration (MF) membranes (Toray, Tokyo, Japan). The membrane was made of polyvinylidene-fluoride (PVDF) and had a nominal pore size of $0.4 \mu \mathrm{m}$. On the other hand, MBR12 and MBR50 are normal submerged MBRs with, respectively, a solid retention time of 12 and 50 days. Hollow-fiber MF membranes (Mitsubishi Rayon Engineering, Tokyo, Japan) made from PVDF polymer were used in MBR12 and MBR50. Nominal pore size of the membranes was $0.4 \mu \mathrm{m}$. Table 1 summarizes the details and operational conditions of the three MBRs.

NF/RO: Permeate of MBR18 was treated using a parallel set of nano-filtration (NF) and reverse osmosis (RO) (Figure 2). The main pilot system equipped with two separate membrane filtration modules with two 2-inch spiral-wound configurations: one NF membrane module (LES 90, nittouDenkou, Tokyo, Japan) and one RO membrane (ES10, NittouDenkou, Tokyo, Japan). Membrane characteristics of NF/RO membranes are given in Table 2. Membrane flux and recovery, in the continuous operation, were fixed at $460 \mathrm{Lm}^{-2}$ day and $70 \%$ respectively. Permeate samples were collected after one day of operation.

The unit was operated for 6 weeks. No chemical cleaning or back washing was performed. During operation, changes in permeates endotoxin activity and trans-membrane pressure (TMP) were monitored.

\section{Assays}

Endotoxin presence in the samples was assessed using the Limulus amoebocyte lysate (LAL) test. The LAL assay is exquisitely sensitive to endotoxin, and the use of endotoxin-free glassware and plastic ware (including tubes and pipette tips), diluent is compulsory. Results were obtained in endotoxin units (EU), where $10 \mathrm{EU}$ corresponds to $1 \mathrm{ng}$ endotoxin. Commercially available endotoxin standards were used as positive controls and LAL reagent water, water free from endotoxin, was used as negative control.

The dissolved organic carbon (DOC) was measured using a Shimadzu TOC-5000 Total Organic Carbon Analyzer according to analytical standards [10]. A Shimadzu TOC-5000 analyzer is able to perform reproducible accurate and sensitive measurements. Detection limits are as low as $4 \mathrm{ppb}$ and standard deviation is less than $1 \%$ of full scale for the range less than $2000 \mathrm{ppm}$.
Water samples from membrane reactors (mixed liquor) were filtered using $0.45 \mu \mathrm{m}$ filter prior to the measurement.

\section{Results and Discussion}

\section{Continuous operation of MBR18}

MBR18 unit was operated continuously for 6 six weeks. No chemical cleaning or back washing was performed. During operation, changes in feed water and permeates' endotoxin activity, DOC and trans-membrane pressure (TMP) were monitored. DOC and endotoxin values give an idea about the level of organic matter showing endotoxicity, while TMP is a translation of the fouling level. For instance high TMP associated with high organic matter showing endotoxicity, would mean that fouling is occurring due to microbial by-products.

Figure 3 shows that the dissolved organic carbon in the feed water is oscillating. The DOC of permeate is slightly increasing. The TMP data (Figure 5) reveal that fouling is slowly and gradually occurring on the membrane. This indicates that bio-film is slowly and gradually formed on the surface of membrane. Endotoxin concentration of permeate was also measured. The value of endotoxin concentration in both the feed water and permeate is illustrated in Figure 4 . While, this concentration is fluctuating in the feed water, it increased slightly in permeate water from about $600 \mathrm{EU} / \mathrm{ml}$ to about $1000 \mathrm{EU} / \mathrm{ml}$. After twenty days of operation, the endotoxin concentration in permeate is around 1000

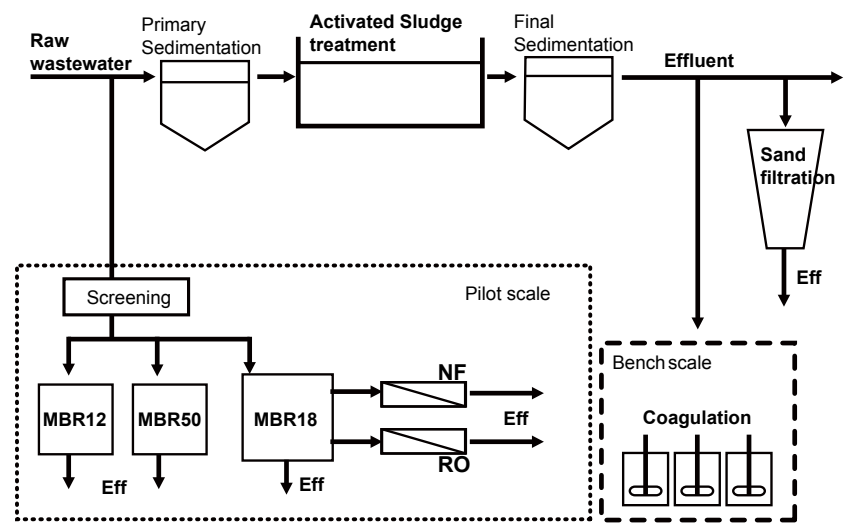

Figure 1: Schematic diagram of the MBR pilot scale treatment system.

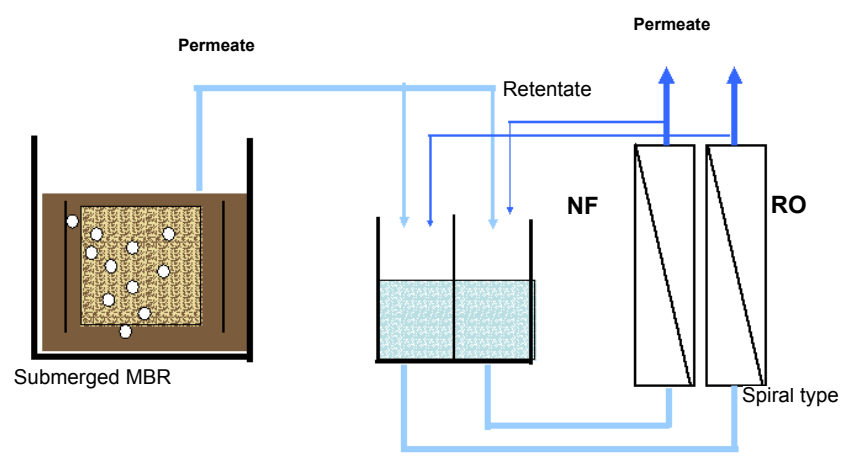

Figure 2: Schematic diagram of the pilot unit MBR18 followed by a paralle set of NF and RO. 
$\mathrm{EU} / \mathrm{ml}$. The increase is associated to the increase of TMP. Two reasons might explain this increase in endotoxin concentration in the MBR permeates. First, Bio-film can be formed on the surface of MBR and it contributes to the release of some endotoxin material (especially low molecular weight MW) that passes through MBR. Second, endotoxic active material is mainly composed of large molecules as reported by Guizani et al. [8]. These large molecules are being biodegraded on the surface of MBR and are converted into smaller molecules that could pass through the membrane.

\section{SRT effect}

The pilot plant, subject of this study, is equipped with three different MBRs, endotoxin was measured in membrane permeates and in membrane reactors of the three MBRS. Figure 6 illustrates endotoxin level in both permeates and reactors. Comparing SRT12, SRT18 and SRT50, at longer SRT the endotoxin and DOC levels in the reactors are the highest (Figure 6 and Table 3). Holakoo et al. [11] reported that longer SRT might lead to the accumulation of higher MW fractions of biomass decay associated soluble microbial products (SMP). Bin and Shuangying [12], using sequencing batch MBR, reported that high MW components become more evident at long SRT. Holakoo et al. [11] concluded that longer SRT might lead to the accumulation of higher MW fractions ( $>100 \mathrm{kDa})$ of biomass-decay-associated SMP. Hence, smaller endotoxin molecules at shorter SRT pass through the membrane. It suggests that in shorter SRT (SRT12), permeates show higher endotoxicity and DOC concentration as compared to other MBRS (Table 3 and Figure 6). At longer SRT, larger molecules and aggregates are formed and cannot pass through the MBR pores, thus showing less endotoxicity and DOC concentration in their permeates. These aggregates increase the endotoxicity and DOC concentration in mixed liquor as shown in Figure 6 and Table 3

Similarly, Guizani et al. [2] applied heat shock protein (HSP) assays to the membrane permeates. The HSP is a very sensitive measurement (bio-assay test) of stress response in cells exposed to pollutants. They found that no significant stress response was detected in the MBR permeates except MBR12, which was operated under a shorter SRT (12 days). It means that at SRT18 and SRT50, toxic molecules could not pass through the membrane. Hence, it is thought that biological organic matter and endotoxin rejected by membrane are being accumulated in the reactors.
To study the effect of SRT on stress response, HSP assays were applied to the supernatant of mixed liquor of the two MBRs with SRT50 and SRT12, respectively [2]. The supernatants showed significant stress response, and in both cases, the stress was significant in samples including higher MW fractions ( $>0.1 \mathrm{um}$ ). The study reports that in the SRT12 case, stress response was significant at all MW ranges. In comparison, the DOC concentration $(<0.45 \mathrm{~mm})$ of MBR12 was higher than MBR50. Holakoo et al. [11] concluded that longer SRT might lead to the accumulation of higher MW fractions (>100 kDa) of biomassdecay-associated SMP. Using sequencing batch MBR, Bin et al. [12] reported that high MW components become more evident at long SRT. MBR operation at shorter SRT induced inadequate biodegradation of the toxic organic matter and resulted in toxicity of all MW fractions. Therefore, in the case of MBR12 the MBR permeate showed a stress response because smaller MW fractions carrying toxicants pass through the membrane. The toxic compounds in a supernatant of mixed liquor cannot be removed at longer SRT, but, toxicity was not detected in permeates. This is probably caused by the fact that small molecules aggregated together into larger molecules and were then removed by MBR. Knowing that the detected toxicity was somehow correlated to the existence of microbial by-products and precisely LPS endotoxin [2], therefore we can conclude that LPS endotoxin can be removed by MBR and the removal is enhanced by their aggregation into larger molecules. However, selection of the pore size of the membrane will be important because the size (MW) of organic matter and the aggregates, showing endotoxicity, are strongly dependent on SRT.

\section{Endotoxin removal using NF and RO}

In MBR18 and MBR 50, large aggregates were formed and this has led to a lower endotoxicity in permeate. In general, at longer SRT, better efficiency of MBR is expected. However longer SRT will induce more cost for treatment. Therefore for a better optimization, NF/RO unit is tested with a permeate water from MBR operated at a moderate SRT (SRT18). It might be interesting that further studies investigate the optimization of MBR-NF/RO treatment, to study at which SRT we can get better treatment at low cost and with less fouling.

The MBR18 permeate was treated using NF and RO. The DOC, TMP and endotoxin were measured. Dissolved organic carbon was below detection limits in NF and RO permeates. The NF/RO system removed a significant amount of the remaining endotoxin

\begin{tabular}{|c|c|c|c|c|c|}
\hline ID & MBR Type & & $\begin{array}{l}\text { Volume } \\
\mathrm{m}^{3}\end{array}$ & $\begin{array}{l}\text { Nominal pore size } \\
\mu \mathrm{m}\end{array}$ & $\begin{array}{l}\text { SRT } \\
\text { Days }\end{array}$ \\
\hline MBR50 & Submerged & Hollow fiber & 0.00255 & 0.4 & 50 \\
\hline MBR18 & Baffled-submerged & Flat sheet & 0.712 & 0.4 & 18 \\
\hline MBR12 & Submerged & Hollow fiber & 0.00255 & 0.4 & 12 \\
\hline
\end{tabular}

Table 1: Characteristics of the three MBRs.

\begin{tabular}{|lllll|}
\hline ID & Membrane material & $\begin{array}{l}\text { Zeta potential } \\
\mathbf{m V}\end{array}$ & $\begin{array}{l}\text { Water permeability } \\
\mathrm{Lm}^{-2} \text { day }^{-1} \mathrm{Kpa}^{-1}\end{array}$ & $\begin{array}{l}\text { Salt rejection } \\
(\%)\end{array}$ \\
\hline NF(LES90) & Polyamide & -8.6 & 1.6 & 90 \\
RO(ES10) & Polyamide & -15.3 & 1.2 & $\mathbf{8 0}$ \\
$\mathbf{8 0}$ & 95.5 \\
\hline
\end{tabular}

Table 2: Characteristics of NF and RO membranes.

\begin{tabular}{|c|c|c|c|}
\hline & DOC (mg & & \\
\hline & MBR50 & MBR18 & MBR12 \\
\hline Feed water & 21 & 21 & 21 \\
\hline Permeate & 4 & 3.8 & 4.5 \\
\hline Mixed liquor (supernatant) & 15.1 & 15 & 8.5 \\
\hline
\end{tabular}

Table 3: DOC concentration of feed water, permeate and of the mixed liquor of the three MBRs. 


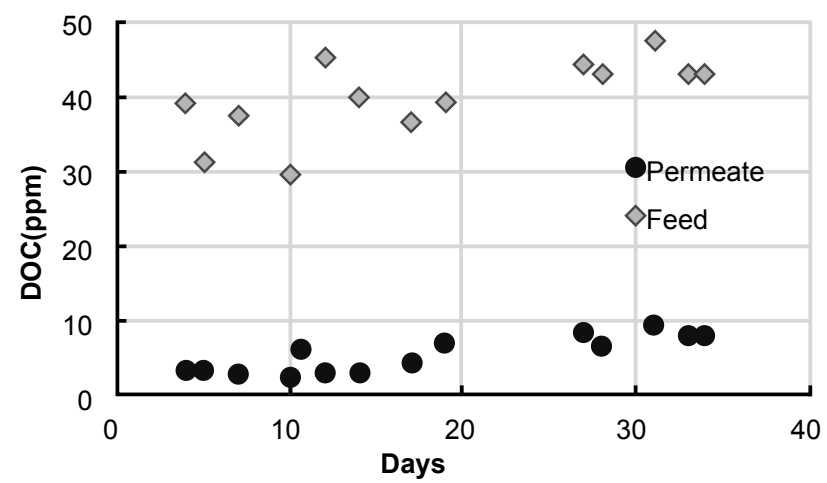

N.B.: There is no preference in choosing the membrane material. We used the polyamide thin film composites, simply because it is a Common membrane material. It is recommended that further studies focus on the effect of membrane material on endotoxin removal.

Figure 3: Dissolved organic carbon (DOC) concentration in MBR18.

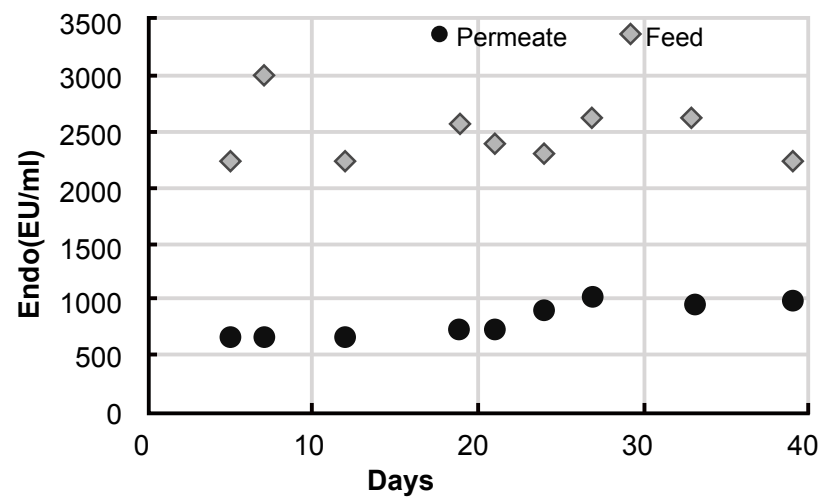

Figure 4: Endotoxin concentration in MBR18.

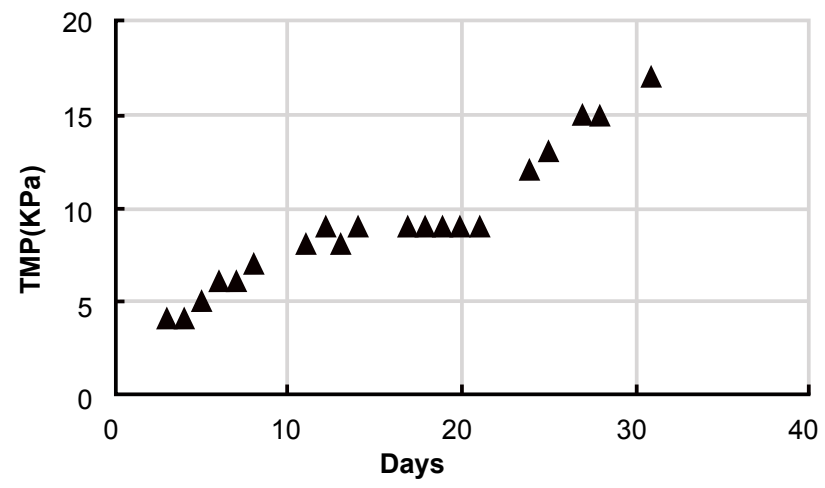

Figure 5: Trans-Membrane Pressure (TMP) in MBR18.

concentration in the MBR permeates. The endotoxin concentration, as illustrated in Figure 7, is lower than $100 \mathrm{EU} \cdot \mathrm{mL}^{-1}$ but still higher than tap water level. Endotoxin concentration in tap water ranges from 1 EU.mL ${ }^{-1}$ to 50 EU.mL ${ }^{-1}$ [13]. The endotoxin concentration in NF/ RO permeates increases as a function of time. Even though, TMP of
$\mathrm{NF} / \mathrm{RO}$ is increasing (Figure 8), it is not well understood whether this increase in endotoxin concentration is due to biofilm formation or because the endotoxin has increased in the feed water. Feed water in MBRs permeate experiences an increase of endotoxin concentration as discussed above. In addition, a study on stress response to cells exposed to NF and RO permeates reported that endotoxin removal by these membrane units, led to a decrease in the stress response level [2]. We should notice that DOC value in NF and RO membranes were below detection limits.

\section{Discussion}

Investigations on the fate of endotoxin in advanced reclamation processes revealed that the MBR-NF/RO set could lead to a substantial removal of endotoxin. Within the observed period of time, efficiency of removal of MBR-NF/RO has decreased. However, based on the available data on endotoxin in tap water [13], the endotoxin concentration in product water (membrane permeates) was 1.5 to 3 fold of endotoxin
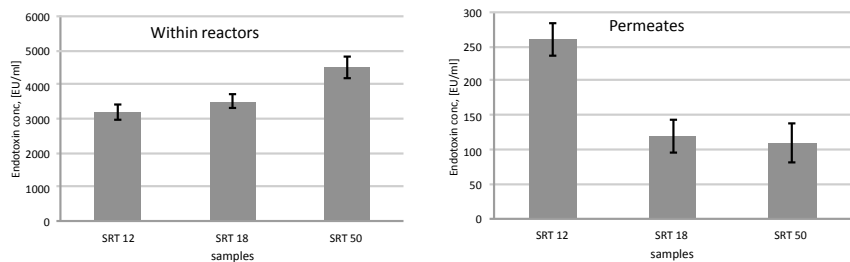

Figure 6: Endotoxicity in membrane permeates and in within the reactors

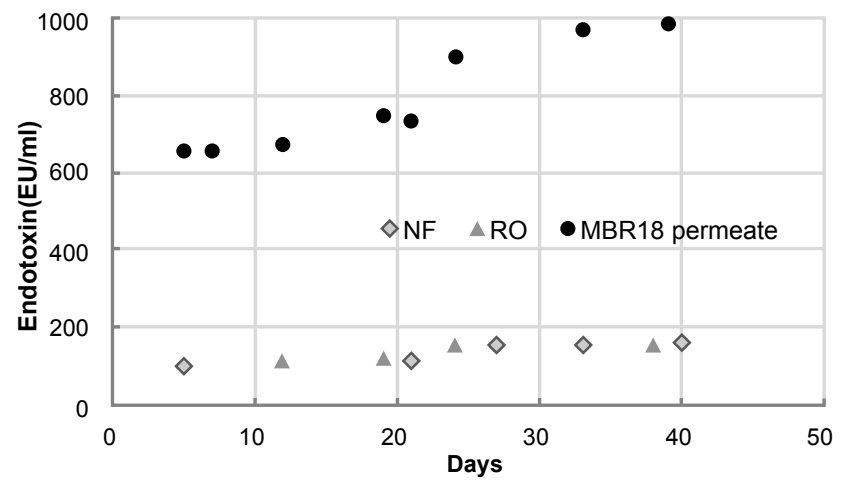

Figure 7: Endotoxin concentration in NF/RO pilot scale.

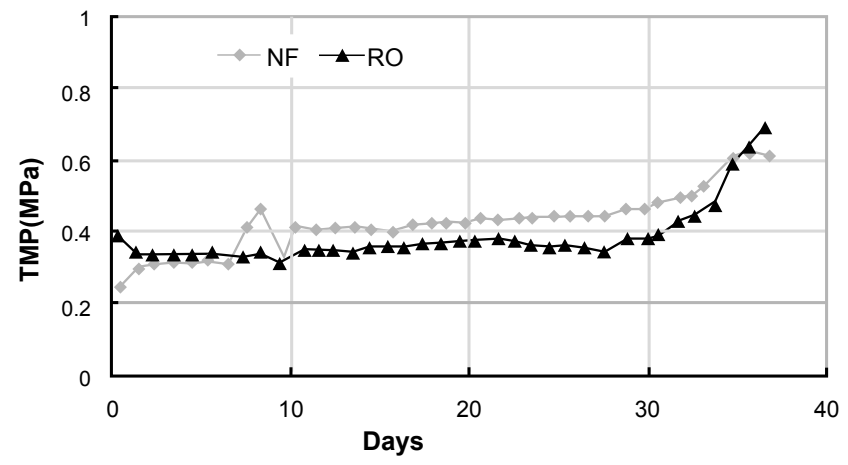

Figure 8: Trans-Membrane Pressure TMP in NF/RO pilot scale. 
Citation: Mokhtar G, Naoyuki F (2012) Microfiltration, Nano-filtration and Reverse Osmosis for the Removal of Toxins (LPS Endotoxins) from Wastewater. J Memb Sci Technol 2:118. doi:10.4172/2155-9589.1000118

Page 5 of 5

present in drinking water.

In the absence of health standards related to allowable endotoxin consumption, and with a final concentration of about $100 \mathrm{EU} \cdot \mathrm{ml}^{-1}$ for NF and RO permeates, subsequent of MBR, it not advisable to reuse these reclaimed waters for direct potable practices as they show a concentration much higher than that present in drinking water. In terms of this study, it becomes obvious that in some cases treated water can be diluted with drinking water at an appropriate proportion. Dilution of recycled water in the environmental buffer also minimizes any potential risk by decreasing the concentration of endotoxins that may be present. In some other cases we need to investigate the effect of storage on the fate of endotoxin. In some extreme cases, further treatments are needed.

\section{Conclusion}

MBR-NF/RO treatment system is effective for endotoxin removal, and this system can be multi-barrier to achieve the stable and high quality of reclaimed water. However, it remains possible that endotoxin removal patterns change depending on combination of treatment or membrane fouling due to long-term operation. Further investigations are needed.

Within the observed period of time, endotoxin concentration in permeates of MB-NF/RO is higher than that of tap water. For a successful reuse practices, two ways can be approached: Dilution of MB-NF/RO permeates with drinking water to reach acceptable concentration, or further treatments are required. Effect of storage of water contaminated with endotoxin is one of the issues to be further investigated.

\section{Acknowledgments}

The authors wish to thank Kimura Katsuki (Assoc. Prof.) as well as wastewater treatment plant operators for cooperation with regard to the pilot unit and assistance. Special thanks are also addressed to Sapporo Sewer Bureau for financial support of this research.

\section{References}

1. Williams LK (2007) Endotoxins: Pyrogens, lal testing and depyrogenation (chap.4: Endotoxin structure, function and activity), CRC Press.

2. Guizani M, Nogoshi Y, Ben Fredj F, Han J, Isoda H, et al. (2012) Heat shock protein 47 stress responses in Chinese hamster ovary cells exposed to raw and reclaimed wastewater. J Environ Monit 14: 492-498.

3. Hindman SH, Favero MS, Carson LA, Peterson NJ, Schonberger LB, et al (1975) Pyrogenic reactions during haemodialysis caused by extramural endotoxin. Lancet 2: 732-734

4. Muittari A, Kuusisto P, Virtanen P, Sovija.rvi A, Gro.nroos P (1980) An epidemic of intrinsic allergic alveolitus caused by tap water. Clin Allergy 10: 77-90.

5. Rylander R, Haglind P, Lundholm M, Mattsby I, Stenqvist K (1978) Humidifier fever and endotoxin exposure. Clin Exp Allergy 8: 511-516.

6. Guizani M, Dhahbi M, Funamizu N (2009) Assessment of endotoxin activity in wastewater treatment plants. J Environ Monit 11: 1421-1427.

7. Guizani M, Dhahbi M, Funamizu N (2009) Survey on LPS endotoxin in rejected water from sludge treatment facility. J Environ Monit 11: 1935-1941.

8. Guizani M, Yusuke N, Dhahbi M, Funamizu N (2011) Characterization of endotoxic indicative organic matter (2-keto-3deoxyoctulosonic acid) in raw and biologically treated domestic wastewater. Water Res 45: 155-162.

9. Li L, Luo RG (1999) Quantitative determination of $\mathrm{ca}^{2+}$ effects on endotoxin removal and protein yield in a two-stage ultrafiltration process. Sep Sci Technol 34: $1729-1741$.

10. American Public Health Association (1985) Standard Methods for the Examination of Water and Wastewater, 16th edn. Washington, DC.

11. Holakoo L, Nakhla G, Yanful EK, Bassi AS (2006) Chelating properties and molecular weight distribution of soluble microbial products from an aerobic membrane bioreactor. Water Res 40: 1531-1538.

12. Dong B, Jiang S (2009) Characteristics and behaviors of soluble microbial products in sequencing batch membrane bioreactors at various sludge retention times. Desalination 243: 240-250.

13. Anderson WB, Slawson RM, Mayfield Cl (2002) A review of drinking-waterassociated endotoxin, including potential routes of human exposure. Can $\mathrm{J}$ Microbiol 48: 567- 587 